Case

Report

\title{
A Giant Thoracic Duct Cyst as the Cause of Abdomen Pain: A Case Report and Review of the Literature
}

Xinyue Wan, MD, $\mathrm{PhD}$ and Zhongyin Zhou MD, $\mathrm{PhD}$

\begin{abstract}
Thoracic duct cysts, which may be of congenital or degenerative origin, are very rare lesions. Most patients are asymptomatic, but when symptoms are present they include cough, dyspnea, dysphagia and chest pain. However, in this case report a 35-year-old male patient presented to us with intermittent abdomen pain. Clinical symptoms and radiographic findings helped to identify a giant thoracic duct cyst in this patient. Surgical resection of the cyst resolved the abdominal symptoms. This was the first case reported in the literature of a thoracic duct cyst with the symptoms of abdominal pain.
\end{abstract}

Keywords: abdominal pain; mediastinal cyst; thoracic duct

\section{Introduction}

The thoracic duct is the largest lymphatic vessel of the lymphatic system. It originates from the cisterna chyli in the abdomen, traverses the diaphragm at the aortic aperture and penetrates through the posterior and superior mediastinum. Thoracic duct cysts are uncommon entities that are usually found in the thoracic segments of the thoracic duct, although these cysts may occur anywhere along the course of the duct. ${ }^{1)}$ A review of the literature revealed that the complaint of intermittent abdominal pain with thoracic duct cysts has only been reported for one case. In this article, we present a rare case of a thoracic duct cyst that emphasizes the diagnosis and provides a review of the literature.

\section{Case Report}

A 35-year-old man was referred to our department complaining of dull quality, intermittent, upper abdominal

Department of Gastroenterology, Wuhan University, Renmin Hospital, China

Received: November 18, 2014; Accepted: February 17, 2015 Corresponding author: Zhongyin Zhou. Department of Gastroenterology, Wuhan University, Renmin Hospital, Wuhan, No. 238 Jiefang Road, Wuhan 430060, Hubei Province, China

Email: zhouzhongyin@outlook.com

(C)2015 The Editorial Committee of Annals of Thoracic and Cardiovascular Surgery. All rights reserved. pain for the past two months. The location of pain was usually in the epigastric and periumbilical region, and this symptom was aggravated by eating or standing for longer periods of time. The abdominal pain or discomfort was typically relieved by laying flat for one or two hours. Medical history was unremarkable for trauma or surgery. Physical examination and laboratory findings were also normal. An extensive evaluation including upper gastrointestinal endoscopy and colonoscopy failed to reveal any other abnormalities. Finally, the computed tomographic (CT) scan revealed an oval, well-defined, non-enhancing, homogeneous, hypodense cystic lesion in the posterior mediastinum that compressed the esophagus, left lower pulmonary vein, and descending aorta (Fig. 1A). Coronal scan showing a large dilated cyst lesion from the level of right inferior pulmonary vein to the retroperitoneum the level of first lumbar vertebra (Fig. 1B). The average CT density of this lesion was 3-8 hounsfield units. So the radiologic studies led to potential diagnosis of a giant thoracic duct cyst. After we excluded some common causes of abdomen pain, such as cholelithiasis, peptic ulcer, digestive tract tumors and lumbar diseases, then this giant cyst was suspected as the sole cause of pain. The patient opted to undergo an exploratory thoracotomy. After ligation, the cystic tumor was dissected and removed (Fig. 1C). The maximum diameter of the soft cyst was $5 \mathrm{~cm}$, and the thoracic duct entered into the cyst inferiorly and superiorly. The cyst $4.2 \mathrm{~cm} \times 5 \mathrm{~cm} \times 11.4 \mathrm{~cm}$ 


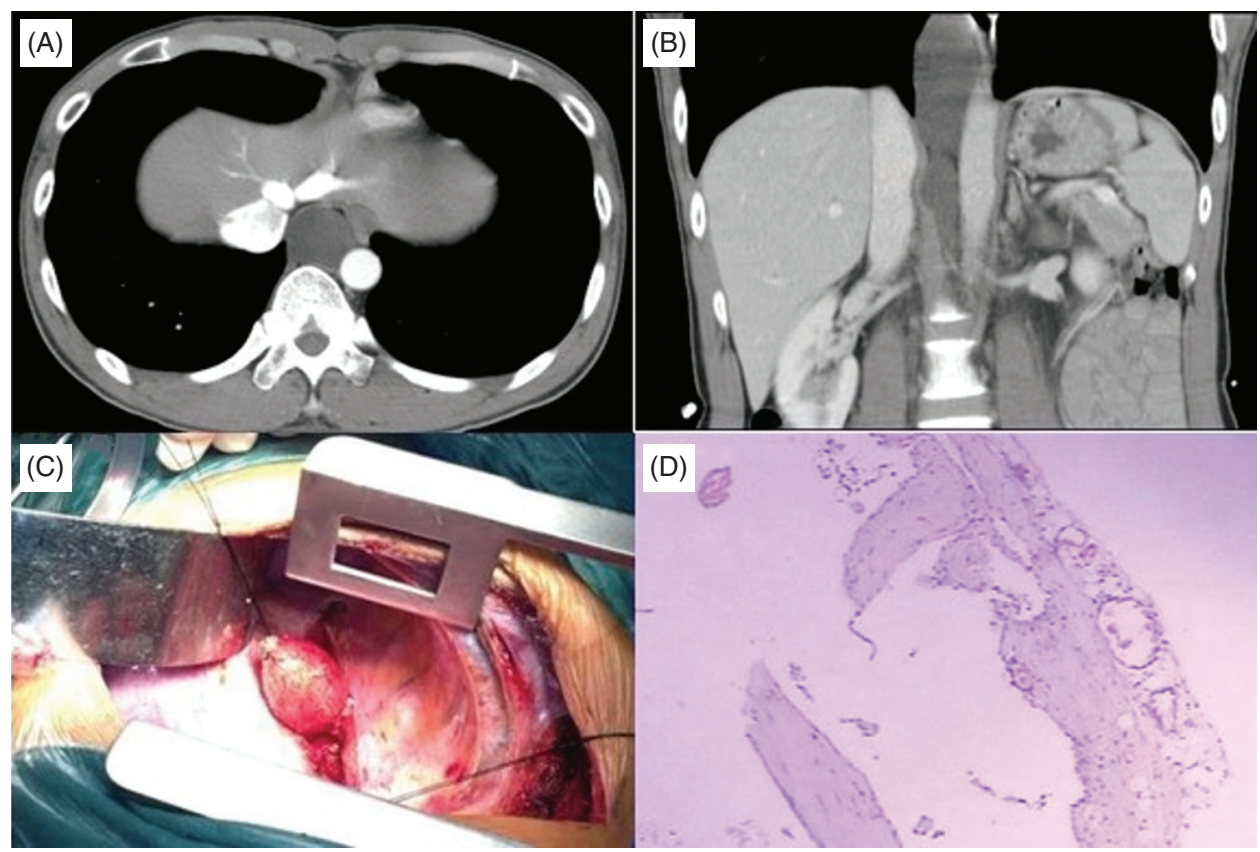

Fig. 1 (A) CT scan showing an oval, homogeneous, non-enhancing cystic mass in the posterior mediastinum. (B) Coronal scan showing a large dilated cyst lesion from the level of right inferior pulmonary vein to the retroperitoneum. (C) Intraoperative findings. (D) The cyst wall consists of fibrous connective tissue and small foci of lymphoid cell aggregation (hematoxylin-eosin, original $\times 4$ ).

contained milky-yellowish chylous fluid, and histological examination of the lesion showed a thin wall lined by a monolayer of flat endothelial cells. Pathological examination confirmed that the cyst was a giant thoracic duct cyst (Fig. 1D). Following uneventful recovery from the operation, the patient remained asymptomatic for 18 months.

\section{Discussion}

Thoracic duct cysts are rare. The first reported case was found during an autopsy examination by Carbone in 1892 and the first antemortem description of this disease was made by Emerson in 1950. ${ }^{2)}$ The exact number of cases is unknown, because the literature describes contradicting results. According to Mattila 30 cases of thoracic duct cysts have been reported since 1999.3) However, in Karajiannis's report published in 2000, the number of cases was 27. ${ }^{4)}$ By 2009, Mortman summarized and reported the number of thoracic duct cysts to be less than $30 .^{5)}$ Because of these contradictory numbers, we decided to make a comprehensive literature review by searching the PubMed database using the keyword "thoracic duct cyst" from the start year up to Aug. 2014. As a result of the review, we found a total of 62 patients with thoracic duct cysts reported previously (30 mediastinal, 31 cervical, and 1 abdominal). The mean age of the patients was 49.0 years (range 17 to 86 years) and 31 patients were female (Table 1).

Although the pathogenesis of the thoracic duct cysts remains unknown, it is thought to be related to the congenital or degenerative weakness of the wall of the thoracic duct and obstruction of the lymphoid flow. ${ }^{5,7)}$ Other causes these duct lesions are iatrogenic injuries and blunt traumas. ${ }^{10)}$ For example, a 29-year-old woman was diagnosed with a cervical thoracic duct cyst after the whiplash injury in a traffic accident 3 months previously. ${ }^{28)}$ Cysts can occur anywhere along the course of the duct, but they are commonly located in the mediastinum (31/63) and neck (31/63). Almost 50\% of the cases were cervical thoracic duct cysts commonly located on the left side. Only one single report described its location on the rightlateral cervical region. ${ }^{3)}$ Thirty-one (49\%) patients with thoracic duct cysts were female, and there is no predominant gender predisposition. Twenty-eight (44\%) patients were asymptomatic. When symptoms were present, they ranged from cough, dysphagia, dyspnea, and chest pain. These were common symptoms because of the pressure 


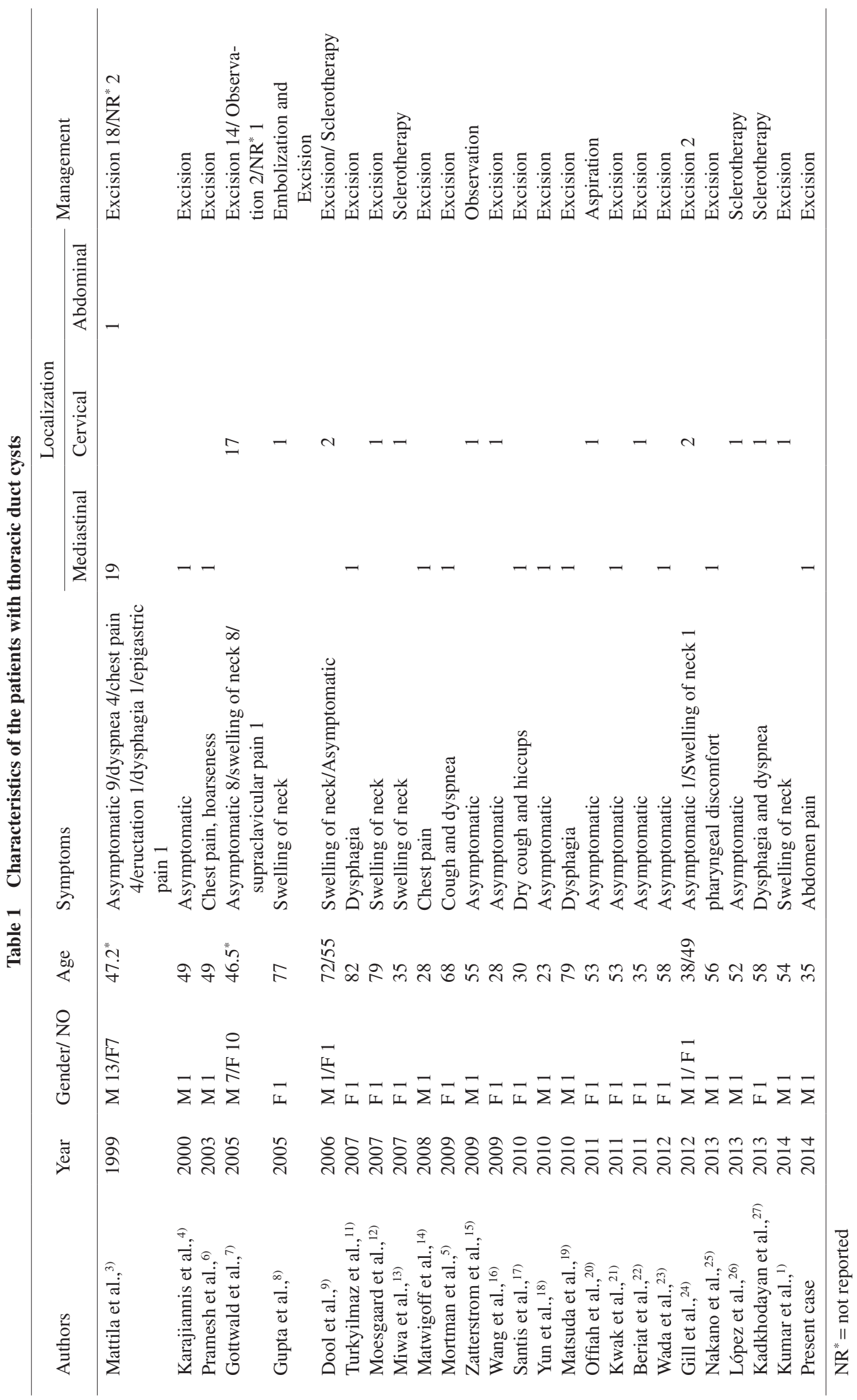


on adjacent structures. ${ }^{6-9)}$ There was a 42 -year-old male patient complaining of sharp left upper quadrant pain occurring 1-2 $\mathrm{h}$ after meal, and relieved by milk and bicarbonate of soda. ${ }^{10)}$ With the help of histodiagnosis after the exploratory thoracotomy, a mediastinal thoracic duct cyst was confirmed. However the patient's original abdominal symptoms still persisted after the surgery. The character of the abdominal pain was more like ulcer pain, and gastroscopy was not performed. For this reason there is doubt about the etiology of the abdominal pain. In this present case report, the initial differential diagnosis of a previously healthy young man with abdominal pain was broad. Some common causes were excluded by history, physical examination and other routine examinations. With the help of CT we found the giant cystic lesion, but we could not determine that this was the cause of our patient's symptoms. Given the size of the giant cystic lesion and characteristics of abdominal pain, we concluded that cystic pressure often increased by food intake or standing for longer periods of time. This, in turn, compressed the adjacent viscera, which most likely contributed to the intermittent abdominal pain of this patient. Thus, we concluded this large cyst was the etiology of abdominal pain and discomfort, and surgical resection was performed to clear the cystic lesion. After the operation, his abdominal pain was resolved completely.

The preoperative diagnosis of thoracic duct cysts is difficult. Magnetic resonance imaging (MRI), especially T2-weighted images, is excellent for demonstrating the anatomic boundaries and the cystic nature of lesions. However, this imaging is less helpful to differentiate thoracic duct cysts from other cystic lesions, such as pericardial or pleural mesothelial cysts, bronchial/esophageal duplication cysts, or neuroenteric cysts. ${ }^{12)}$ The lymphangiograms was thought as the "gold standard" of thoracic duct imaging, ${ }^{4)}$ but this technology now is used less and less in clinical. The endoscopic ultrasound guided fine needle tissue acquisition (EUS-FNA) system has led to the development of a better method, especially for diagnosing mediastinal thoracic duct cysts. EUS-FNA system is excellent for identifying lymphoceles and showing anechoic or hypoechoic masses. The fine-needle aspiration can reveal a milky-white or brown fluid, and this is helpful for the biochemical analysis. In addition, histopathologic analysis can be used to confirm the presence of lymphocytes that will yield strong evidence for thoracic duct cysts. Wada et al. used the EUS-FNA system successfully to demonstrate a $4 \mathrm{~cm} \times 2 \mathrm{~cm} \times 1 \mathrm{~cm}$ mediastinal thoracic duct cyst before the surgery, this procedure was safely performed without complication. ${ }^{23)}$ We tried to perform EUS-FNA to get a more accurate diagnosis before the surgery for our patient. But the patient was concerned about the complications about this procedure, so he opted for the surgery.

Surgical resection of the cyst is usually recommended; however, direct puncture sclerotherapy was reported to be an alternative method, ${ }^{9,13)}$ the long term effect still need to be evaluated. In fact most of the patients received more active therapy either with surgery or sclerotherapy. Fifty-two patients chose the excision; four patients chose the sclerotherapy; four people chose the conservative method. Postoperative chylothorax was the most common complication and happened in five patients, four of which had to receive re-operation. Among the four patients who received the sclerotherapy, one patient had a fever for 5 days after the treatment. ${ }^{13)}$ Because cysts of the thoracic duct typically grow slowly and the symptoms are usually due to compression of the adjacent structures, treatment for the relief of the compression is critical. One report advocated initial conservative management by needle aspiration and a low-fat diet supplemented with mediumchain triglyceride oil. ${ }^{29)}$ Also Zätterstrom et al. followed a 55 year old male with cervical cystic dilatation and widened intrathoracic duct for 25 years without intervention. ${ }^{15)}$ In both of these cases the cysts underwent remission. Therefore while symptomatic lesions should be resected, it is better to simply follow up in asymptomatic patients with confirmed diagnosis over time. Thoracic duct cysts are benign lesions. No cases of malignant transformation has been reported. ${ }^{1,3)}$ So surgery is optional for the patient with a small and asymptomatic cyst.

In summary, thoracic duct cysts are rare lesions and abdominal pain should be considered as one of its symptoms. Thoracic duct cyst is difficult to diagnose before the surgery. Endoscopic ultrasound guided fine needle tissue acquisition could better identify the cyst location and the relationship with adjacent structure. In addition, aspiration of chylous fluid within cysts assists the diagnosis of thoracic duct cysts. Surgical resection is the basic modality of treatment for symptomatic patients; whereas, a more conservative approach is advisable for the asymptomatic patients.

\section{Disclosure Statement}

There was no fund support of this report and no conflict of interest was declared by the authors. We thank Farber Jay for his editorial assistance in writing this manuscript. 


\section{References}

1) Kumar A, Ramakrishnan TS, Sahu S. Primary cervical thoracic duct cyst: a case report and review of the literature. Ear Nose Throat J 2014; 93: E17-21.

2) Emerson GL. Supradiaphragmatic thoracic-duct cyst - An unusual mediastinal tumor. N Engl J Med 1950; 242: 575-78.

3) Mattila PS, Tarkkanen J, Mattila S. Thoracic duct cyst: a case report and review of 29 cases. Ann Otol Rhinol Laryngol 1999; 108: 505-8.

4) Karajiannis A, Krueger T, Stauffer E, et al. Large thoracic duct cyst - a case report and review of the literature. Eur J Cardiothorac Surg 2000; 17: 754-6.

5) Mortman KD. Mediastinal thoracic duct cyst. Ann Thorac Surg 2009; 88: 2006-8.

6) Pramesh CS, Deshpande MS, Pantvaidya GH, et al. Thoracic duct cyst of the mediastinum. Ann Thorac Cardiovasc Surg 2003; 9: 264-5.

7) Gottwald F, Iro H, Finke C, et al. Thoracic duct cysts: a rare differential diagnosis. Otolaryngol Head Neck Surg 2005; 132: 330-3.

8) Gupta M, Lovelace TD, Sukumar M, et al. Cervical thoracic duct cyst. J Thorac Imaging 2005; 20: 107-9.

9) Dool JJ, de Bree R, van den Berg R, et al. Thoracic duct cyst: sclerotherapy as alternative for surgical treatment. Head Neck 2007; 29: 292-5.

10) BAKST AA. Blind supradiaphragmatic thoracic duct cyst; case report. Ann Surg 1954; 140: 250-3.

11) Turkyilmaz A, Eroglu A. A giant thoracic duct cyst: an unusual cause of dysphagia. J Thorac Cardiovasc Surg 2007; 134: 1082-3.

12) Moesgaard L, Baerentzen S, Mirz F. Cervical thoracic duct cyst: a differential diagnosis of left supraclavicular swelling. Eur Arch Otorhinolaryngol 2007; 264: 797-9.

13) Miwa T, Tatsutomi S, Tsukatani $T$, et al. OK-432 therapy for a cervical thoracic duct cyst. Otolaryngol Head Neck Surg 2007; 136: 852-3.

14) Matwiyoff GN, Bradshaw DA, Hildebrandt KH, et al. A 28-year-old man with a mediastinal mass. Thoracic duct cyst. Chest 2008; 134: 648-52.

15) Jackson AS, Reinsberg SA, Sohaib SA, et al. Dynamic contrast-enhanced MRI for prostate cancer localization. Br J Radiol 2009; 82: 148-56.
16) Wang YA, Zhang ZY, Zheng JW, et al. Spontaneous thoracic duct cyst presenting as a left supraclavicular mass - report of a case and review of literature. Phlebology 2009; 24: 82-4.

17) De Santis M, Martins V, Fonseca AL, et al. Large mediastinal thoracic duct cyst. Interact Cardiovasc Thorac Surg 2010; 10: 138-9.

18) Yun JS, Oh SG, Song SY, et al. Mediastinal thoracic duct cyst adjacent to left pericardium. Eur J Cardiothorac Surg 2011; 39: 274.

19) Matsuda E, Okabe K, Yamamoto H, et al. [Pericardial cyst that involved thoracic duct]. Kyobu Geka 2010; 63: 470-3.

20) Offiah CE, Twigg S. Lymphocoele of the thoracic duct: a cause of left supraclavicular fossa. Br J Radiol 2011; 84: e27-30.

21) Kwak MY, Bae CH. Thoracic Duct Cyst in Mediastinum - A case report -. Korean J Thorac Cardiovasc Surg 2011; 44: 83-5.

22) Beriat GK, Kocatürk S, Demirdağ M, et al. Supraclavicular thoracic duct cyst. Kulak Burun Bogaz Ihtis Derg 2011; 21: 159-62.

23) Wada H, Yoshida S, Ishikawa A, et al. Endobronchial ultrasonography in a patient with a mediastinal thoracic duct cyst. Ann Thorac Surg 2012; 93: 1722-5.

24) Gill MT, Lian TS, Thibodeaux JD, Nathan CA. Cervical thoracic duct cyst: importance of preoperative suspicion for appropriate management of left-sided neck mass. Ear Nose Throat J 2012; 91: E13-15.

25) Nakano T, Okamoto H, Maruyama $S$, et al. Threedimensional imaging of a thoracic duct cyst before thoracoscopic surgery. Eur J Cardiothorac Surg 2014; 45: 585 .

26) Rosique López L, Rosique Arias M. [Thoracic duct cyst, sclerosing treatment]. Acta Otorrinolaringol Esp 2014; 65: 211-3.

27) Kadkhodayan Y, Yano M, Cross DT 3rd. Direct puncture sclerotherapy of a thoracic duct cyst presenting as an enlarging left supraclavicular mass. BMJ Case Rep 2013; 30 .

28) Mosahebi A, Gleeson M, Owen WJ. Mass in the neck after whiplash injury. J R Soc Med 1998; 91: 493-4.

29) Van den Bussche D, Audenaert E, De Gendt G, et al. Conservative treatment of a cervical thoracic duct cyst: a case report. Acta Chir Belg 2007; 107: 67-9. 